\title{
FETAL CYSTIC HYGROMA ASSOCIATED WITH TERMINAL 2p25.1 DUPLICATION AND TERMINAL 3p25.3 DELETION: CYTOGENETIC, FLUORESCENT IN SITU HYBRIDIZATION AND MICROARRAY FAMILIAL CHARACTERIZATION OF TWO DIFFERENT CHROMOSOMAL STRUCTURAL REARRANGEMENTS
}

Stipoljev $\mathrm{F}^{1,2, *}$, Barbalic $\mathrm{M}^{3}$, Logara $\mathrm{M}^{3}$, Vicic $\mathrm{A}^{1}$, Vulic $\mathrm{M}^{4}$, Zekic Tomas $\mathrm{S}^{5}$, Gjergja Juraski R $\mathrm{R}^{2,6}$

${ }^{*}$ Corresponding Author: Feodora Stipoljev, Ph.D., Associate Professor, Cytogenetic Laboratory, Department of Obstetrics and Gynecology, Clinical Hospital "Sveti Duh," Sveti Duh 64, 10000 Zagreb, Croatia. Tel.: +385-1371-2273. Fax:+385-1374-5534. E-mail: stipoljev@yahoo.com

\begin{abstract}
We report a prenatally diagnosed case of partial trisomy $2 \mathrm{p}$ and partial monosomy $3 \mathrm{p}$, resulting from unbalanced translocation $(2 ; 3)(\mathrm{p} 25.1 ; \mathrm{p} 25.3)$ of paternal origin. Parents were non consanguineous Caucasians, with familial history of recurrent miscarriages on the father's side. Detailed sonographic examination of the fetus showed a septated cystic hygroma measuring $6 \mathrm{~mm}$ at 13 weeks' gestation. Karyotyping and fluorescent in situ hybridization (FISH) analysis of cultured amniotic fluid cells revealed an unbalanced translocation $\operatorname{der}(3) \mathrm{t}(2 ; 3)(\mathrm{p} 25.1$; $\mathrm{p} 25.3)$ and apparently balanced inv(3)(p13p25.3) in a fetus. Parental cytogenetic evaluation using karyotyping and FISH analysis showed the presence of both a balanced translocation and a paracentric inversion in father $\mathrm{t}(2 ; 3)(\mathrm{p} 25.1 ; \mathrm{p} 25.3)$ inv(3)(p13p25.3). Microarray analysis showed a $11.6 \mathrm{Mb}$ deletion at 3p26.3-p25.3 and duplication of $10.5 \mathrm{Mb}$ at the $2 \mathrm{p} 25.3-\mathrm{p} 25$ region. The duplicated region at $2 \mathrm{p} 25.1 \mathrm{p} 25.3$ contains 45 different genes, where 12 are reported as OMIM morbid genes with different phenotypical implications. The deleted region at $3 \mathrm{p} 26.3$ p25.3 contains 65 genes, out of which 27 are OMIM genes.
\end{abstract}

\footnotetext{
Cytogenetic Laboratory, Department of Obstetrics and Gynecology, Clinical Hospital "Sveti Duh," Zagreb, Croatia

${ }^{2}$ Faculty of Medicine, Josip Juraj Strossmayer University of Osijek, Osijek, Croatia

${ }^{3}$ Genom Ltd, Zagreb, Croatia

${ }^{4}$ Department of Gynecology and Obstetrics, Split University Hospital Centre, School of Medicine, University of Split, Croatia

${ }^{5}$ Pathology Department, University Hospital Centre Split, Split, Croatia, School of Medicine, University of Split, Croatia

${ }^{6}$ Srebrnjak Children's Hospital, Zagreb, Croatia
}

Three of these (CNTN4, SETD5 and VHL) were curated by Clingene Dosage Gene Map and were given a high haplo-insufficiency score. Genes affected by the unbalanced translocation could have contributed to some specific phenotypic changes of the fetus in late pregnancy. The application of different cytogenetic methods was essential in our case, allowing the detection of different types of structural chromosomal aberrations and more thorough genetic counseling for future pregnancies.

Keywords: Array comparative genomic hybridization (aCGH); Chromosome 2; Chromosome 3; Molecular karyotyping.

\section{INTRODUCTION}

Pure partial trisomy for the terminal short arm of chromosome 2 is an extremely rare chromosomal aberration, described in only a few reports. Observed phenotypical features included prenatal and postnatal growth retardation, facial dysmorphia, congenital heart disease, genital hypoplasia, long widely spaced fingers/toes, and hypotonia [1-4]. Contrarily, terminal deletion of chromosome $3 p$ is classified as a $3 \mathrm{p} 25$-pter deletion syndrome (MIM 613792), while the associated phenotype depends on exact size and location of the deletion. Characteristic features include low birth weight, trigonocephaly, microcephaly, hypertelorism, micrognathia, ptosis, ear anomalies, hypotonia, mental and growth retardation, whereas polydactyly, congenital heart defects, gastrointestinal and renal anomalies are considered as variable features $[5,6]$.

To date, only one case of unbalanced translocation resulting in partial trisomy $2 p$ and partial monosomy $3 p$ has been reported [7]. Herein, we present a prenatally diagnosed case of partial trisomy $2 \mathrm{p}$ and partial monosomy $3 p$, 
resulting from unbalanced translocation $(2 ; 3)(\mathrm{p} 25.1 ; \mathrm{p} 25.3)$ of paternal origin. As father is the carrier of the reciprocal translocation $2 \mathrm{p} ; 3 \mathrm{p}$ and paracentric inversion of short arm of chromosome 3 , this case emphasizes the importance of using different cytogenetic methods for the purposes of final diagnosis settlement.

\section{CASE REPORT}

A 25-year-old gravida 2, para $0(\mathrm{G} 2 \mathrm{P} 0)$ was referred for ultrasonic evaluation of increased nuchal translucency (NT) thickness, detected during routine first-trimester ultrasound screening at another hospital. Her previous pregnancy ended in spontaneous abortion at 17 weeks' gestation. The parents were non consanguineous Caucasians, while familial history revealed recurrent miscarriages in the husband's family. Detailed sonographic examination showed a septated cystic hygroma measuring $6 \mathrm{~mm}$, and chorionic villus sampling (CVS) was performed at 13 weeks' gestation. Cytogenetic analysis of short- and long-term cultured villi showed a male fetal karyotype with derivative chromosome 3. Subsequent amniocentesis was performed at 17 weeks' gestation. A second-trimester examination was unremarkable, except of discrete nuchal thickness. Parental cytogenetic evaluation using karyotyping and FISH analysis showed apparently balanced translocation and paracentric inversion in father $\mathrm{t}(2 ; 3)(\mathrm{p} 25.1 ; \mathrm{p} 25.3) \operatorname{inv}(3)$ (p13p25.3) [Figure 1(A)]. Dual-color FISH was performed on paternal peripheral blood lymphocytes [Figure 1(B)] and cultured amniotic fluid cells according to the manufacturer's instructions, using whole-chromosome painting probes (wcp2, wcp3; Cytocell Ltd., Cambridge, Cambridgeshire, UK) probes specific for cen-tromeres of chromosome 2 and 3 (D2Z1, D3Z1 Kreatech probes; Leica Biosystems Inc., Buffalo Grove, IL, USA), locus specific 3p25 (PPAR $\gamma$; Kreatech), and subtelomeric probes 2p and 3p (D2S52147, D3S4558 Kreatech probes; Leica Biosystems). Classical cytogenetic and FISH analysis of cultured amniotic fluid cells revealed an unbalanced karyotype $46, X Y, \operatorname{der}(3) \mathrm{t}(2 ; 3)$ (p25.1;p25.3)inv(3) (p13p25.3) pat in the fetus, resulting in partial trisomy $2 p$ and partial monosomy $3 p$. Genomic DNA was isolated from cultured amniocytes using DNeasy ${ }^{\circledR}$ Blood \& Tissue Kit (Qiagen Inc., Valencia, CA, USA) according to the manufacturer's protocol. Array comprehensive genomic hybridization (aCGH) was carried out using SurePrint G3 CGH+SNP $180 \mathrm{~K}$ microarray from Agilent Technologies (Santa Clara, CA, USA). Microarray slide was scanned with G4900DA SureScan Microarray Scanner (Agilent Technologies), and data were analyzed by Cytogenomics 3.0.6.6 software (Agilent Technologies). Microarray analysis confirmed unbalanced structural rearrangement in the fetus, and delineated exact breakpoint sites showing a $11.6 \mathrm{Mb}$ deletion at 3p26.3-p25.3 [arr(hg19)3p26.3p25.3 $(100,389-11,723,086) \times 1)$, and duplication in size of 10.5 Mb at 2p25.3-p25.1 [arr(hg19)2p25.3p25.1(39,193-10,595, 414) $\times 3$ ] [Figure 1(C) and 1(D)]. After extensive genetic counseling, the parents decided to terminate the pregnancy at 21 weeks' gestation.

Autopsy and external measurements of the male fetus revealed a weight of $380.53 \mathrm{~g}$, crown-heel length of 25 $\mathrm{cm}$, crown-rump length of $17.5 \mathrm{~cm}$ and head circumference of $17.9 \mathrm{~cm}$. All the measurements were consistent with $21 / 22$ gestational weeks. The fetus had a normally formed head without overriding of the skull bones. Ears were low-set and posteriorly rotated. Hypertelorism and increased nuchal thickness were noted. Limbs were within normal limits and external genitalia were in accordance with male sex. Internal examination of thoracic cavity revealed normally formed heart, thymus and neck structures. The left lung contained two lobes, while the right lung had incomplete horizontal fissure giving the appearance of undeveloped middle lobe, the oblique fissure was present. Abdominal organs were of normal size and position giving the gestational age. The testicles were located in the abdomen, cut surface showed hemorrhage.

\section{DISCUSSION}

We have presented an extremely rare, prenatally diagnosed case of partial trisomy 2p25.3-p25.1 and partial monosomy 3p26.3-p25.3 of paternal origin. To date, only one study by Chen et al. [7] from 1996 reported a very similar unbalanced translocation involving partial $2 p$ trisomy and partial $3 \mathrm{p}$ monosomy. Both parents were phenotypically normal and the mother was a balanced reciprocal translocation carrier 46,XX, t(2;3)(p25.3;p25). Prenatal sonographic findings included single umbilical artery, shortening of the long bones and hypertelorism, while 10-month follow-up revealed craniofacial dysmorphy, hypotonia, growth and mental retardation. In our case, the fetal karyotype showed an unbalanced translocation with a partial trisomy 2p25.1-pter, partial monosomy 3p25.3pter, and balanced paracentric inversion of chromosome 3 with breakpoint sites in 3p13 and 3p25.3, both of paternal origin. The fetus had hypertelorism, low-set posteriorly rotated ears and cystic hygroma. Father is a carrier of two different structural rearrangements with a common breakpoint in 3p25.3. As the total number of breakpoint sites is three, it cannot be classified as a complex chromosomal rearrangement. The investigation of patients with apparently one type of balanced structural chromosomal abnormality can give unexpected findings of another apparently cryptic balanced rearrangement, which can be overcame with use of FISH analysis. 


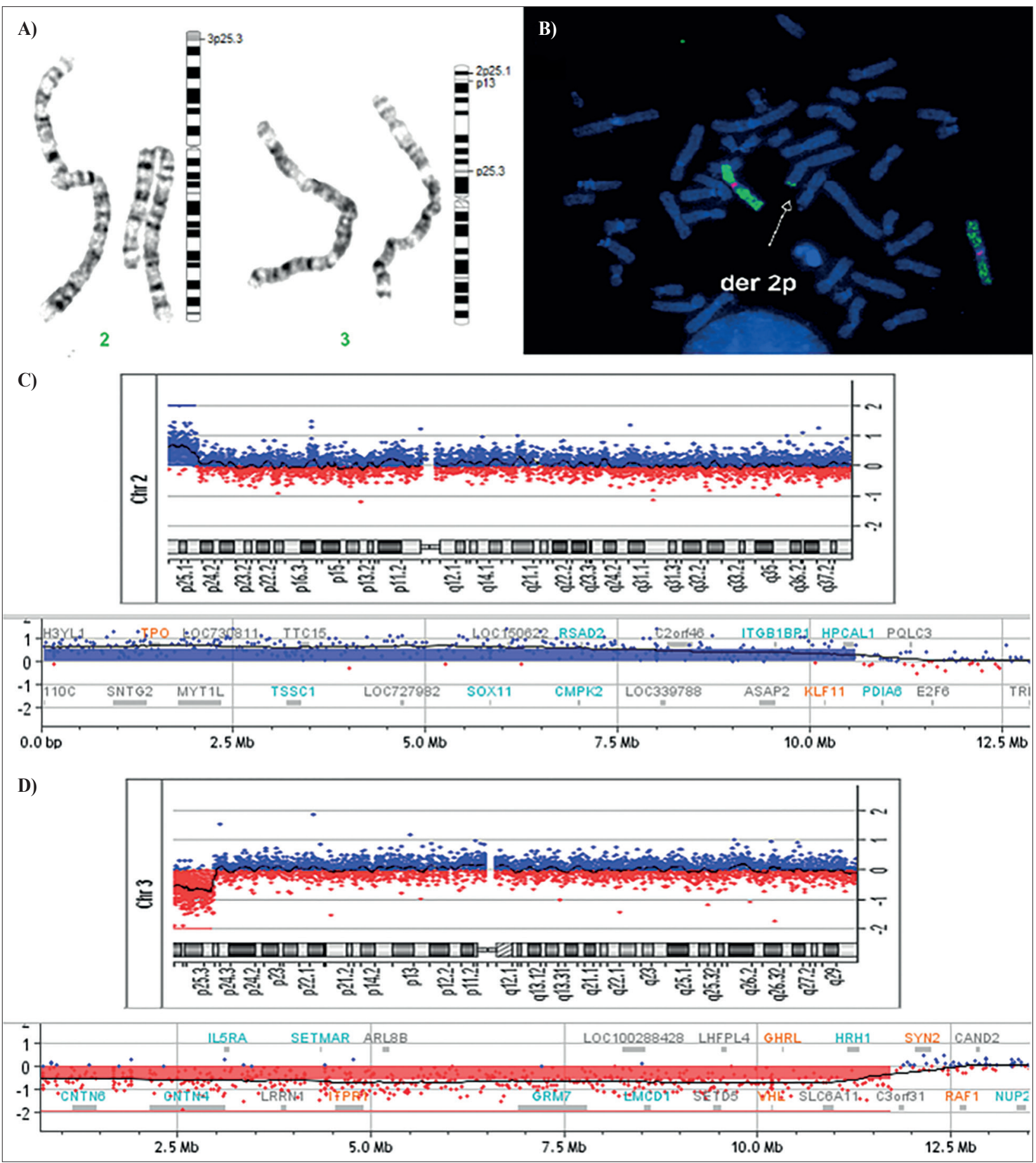

Figure 1. (A) Partial karyotype with accompanied ideograms showing balanced translocation $(2 ; 3)(\mathrm{p} 25.1 ; \mathrm{p} 25.3)$ and paracentric inversion of chromosome 3 in the father. (B) Metaphase FISH on paternal peripheral blood lymphocytes using whole-chromosome painting probe for chromosome 3 (green label) and chromosome 3 specific centromere probe (red label). The arrow indicates derivative chromosome $2 \mathrm{p}$ resulting from $2 p ; 3 p$ reciprocal translocation. (C) Array comprehensive genomic hybridization analysis of cultured amniotic fluid cells: detailed view of 2(p25.1-p25.3) gain. (D) The 3(p25.3-p26.3) deletion (image from Cytoreport generated by Cytogenomics software; Agilent Technologies).

Partial trisomy $2 \mathrm{p}$ has been published in more than 50 cases, mostly in unbalanced translocations with other chromosomes. The main clinical features referring to partial trisomy 2p25.3-p25.1 include developmental delay and craniofacial dysmorphia. Only few studies reported pure duplication of the $2 \mathrm{p}$ terminal region. Wahita et al. [8] described a patient with 2p25.1-pter duplication with trigonocephaly, hypertelorism, anteverted nares, external 
ear anomalies, exophthalmos, arachnodactyly and joint dysplasia, but without developmental delay. Roggenbuck et al. [9] reported a case of 3-year-girl with a significant psychomotor delay and dysmorphy including a prominent forehead, malar hypoplasia, and a prominent nose with a narrow alar base. Additionally, a long torax, a mild pectus carinatum deformity and scoliosisis, with long and slender extremities were found. She had a de novo pure partial trisomy 2p24.3-2pter. Bonaglia et al. [1] reported a familial 10.0 Mb inverted duplication 2p25.3-25.1 and $270 \mathrm{~kb}$ deletion of 2 pter. Two children and their father exhibited mental retardation and craniofacial dysmorphia including hypertelorism, high forehead, low-set and irregular ears, thin upper lip and flat philtrum.

The duplicated 10.5 Mb region $2 \mathrm{p} 25.1 \mathrm{p} 25.3$ in our case contains 45 different genes, where 12 (ODC1, KLF11, RPS7, TPO, MYT1L, PXDN, RNASEH1, COLEC11, SOX11, ADAM 17, KIDINS220, TRAPPC12) are reported as OMIM morbid genes with different phenotypical implications. Coffin-Siris syndrome 9 (CSS9) or autosomal dominant mental retardation-27 (MRD27, MIM 615866) caused by a heterozygous mutation in the SOX11 gene, is usually accompanied by facial dysmorphia, growth deficiency and mild intellectual disability. Tsurusaki et al. [10] emphasized the importance of $S O X 11$, which encodes SoxC group high mobility group (HMG) box-containing transcription factor as a downstream transcriptional factor of the PAX6 and BAF complex in brain development. Moreover, SOX11 and $M Y T 1 L$ were also duplicated in this as in our case. MYT1L is a member of myelin transcription factor 1 family, acting on neuronal differentiation. Disrupted function of one MYT1L allele on 2p25.3 by cryptic gene deletion, intragenic duplication, or point mutation will cause syndromic intellectual disability (mental retardation, autosomal dominant 39 ) in large number of such patients [11]. Bonaglia et al. [1] hypothesized that an increased expression of MYT1L product could lead to developmental difficulties associated with partial $2 \mathrm{p}$ trisomy. $\mathrm{P}$

Lund et al. [12] analyzed 132 fetuses with an increased nuchal translucency of $>3.5 \mathrm{~mm}$ with chromosomal microarray. Among 12 fetuses with pathogenic copy number variants (CNV), one in whom the NT was measured $6.6 \mathrm{~mm}$ had a $46.0 \mathrm{Mb}$ duplication of region 2p25.3-p21. Mother was a balanced translocation carrier. Lee et al. [3], described a fetus with cystic hygroma and unbalanced karyotype with additional chromosomal material on short arm of chromosome 9, designated as distal part of short arm of chromosome 2. Aviron-Goldring et al. [2] described a prenatally diagnosed case of partial trisomy 2pter resulting from an unbalanced karyotype $46, X Y, \operatorname{der}(21) \mathrm{t}(2 ; 21)(\mathrm{p} 24 ; \mathrm{p} 11.1)$ of de novo origin. The pregnancy was terminated at 24 weeks' gestation, and autopsy revealed high forehead, hypertelorism, small nose with depressed nasal bridge, thin upper lip, epicantal folds, micrognatia, low-set slightly rotated ears, short neck with excess skin, proximally placed thumbs, and urogenital anomalies (mildly distended ureters and enlarged kidneys, hypoplastic testes). Thangavelu et al. [13] reported a prenatal case with a terminal duplication 2p25.3-p22 and anencephaly at 16.7 weeks' gestation. Marlet et al. [14] described a prenatal case of de novo inverted duplication 2 p21p25.3 size of $43.75 \mathrm{Mb}$ with present interstitial telomeric sequences at the breakpoint 2 p21 and a tetralogy of Fallot. Nonspecific ultrasound findings described in previous reports regarding prenatal findings in partial $2 p$ trisomy included increased NT/cystic hygroma, as in our case, as well as heart defect, anencephaly, hypertelorism, low-set ears, and urogenital anomalies.

Since the first case in 1978 [5], about 50 cases of the 3pter-p25 contiguous gene deletion syndrome (MIM 613792 ) have been reported. The 11.6 Mb deletion in our case contained 65 genes, out of which 27 are OMIM genes such as CNTN4, SETD5, VHL CHL1 SLC6A1 ITPR1, HRH1, ATG7, CRELD1 and MTMR14. Three of these (CNTN4, SETD5 and VHL) were curated by Clingene Dosage Gene Map (retrieved from www.ncbi.nlm.nih.gov/ projects/dbvar/clingen) and were given a high haploinsufficiency score. The SETD5 (SET domain containing 5, MIM 615743 ) gene encodes a histone methyltransferase highly conserved and expressed in the fetal brain. Heterozygous mutations of SETD 5 cause the autosomal dominant mental retardation 23 (MIM 615761) with different dysmorphic features [15]. Mattioli et al. [16] compared three groups of patients; those who had disrupted only BRPF1, both BRPF1 and SETD5, and only SETD5. The authors noted that in patients with disruption of both genes the intellectual disability was more severe, compared to either of these genes. They concluded that BRPF1 haploinsufficiency, as well as the SEDT5 significantly contributed to syndromic intellectual disability (MIM 617333) in a 3p25-phenotype. CNTN4 encodes a member of the immunoglobulin superfamily of neuronal cell adhesion molecules (Contactin 4, MIM 607280) and has a major role in brain development. Fernandez et al. [17] reported a patient with phenotypical features of $3 p$ deletion syndrome which had de novo balanced translocation $\mathrm{t}(3 ; 10)(\mathrm{p} 26 ; \mathrm{q} 26)$. This translocation disrupted the CNTN4 gene on the 3p26 region and caused growth retardation, development delay and dysmorphic features. Suzuki-Muromoto et al. [18] reported a patient showing classical phenotypical features of $3 p$ deletion but also exhibiting cerebellar hemangioblastoma, which was diagnosed as VHL syndrome. Array comprehensive genomic hybridization analysis revealed a de novo deletion of the 3 p25 region encompassing $V H L$. 
Neurodevelopment difficulties are often present in $3 p$ deletion syndrome. Deletion or disruption of CHL1, ITPR 1 and SLC6A1 genes could lead to neurodevelopmental difficulties because of their high expression patterns in the brain [19-22]. In approximately one-third of patients, a congenital heart defect (CHD), commonly atrioventricular septal defect, has been reported. Shuib et al. [23] reported a detailed aCGH analysis of 14 patients with $3 p$ deletion syndrome. As well as the typical symptoms of $3 p$ deletion syndrome including neurodevelopment delay and different structural malformations, five patients also exhibited CHD. The authors suggested a candidate critical region for CHD of approximately $200 \mathrm{~kb}$ in the region $3 \mathrm{p} 25$ containing genes HRH1 and ATG7, although the involvement of the previously proposed CRELD1 gene [24] located distally could not be excluded. Our case had deleted HRH1, ATG7 and $C R E L D 1$ but without heart defects suggesting variant penetrance in different cases.

CAV3 and MTMR14 were also suggested to contribute to phenotype of $3 p$ deletion syndrome $[25,26]$. CAV3 (Caveolin 3, MIM 601253) and MTMR14 (Myotubularin-related protein 14, MIM 611089) that encodes a muscle-specific inositide phosphatase, contribute to the development and regulation of muscles. Disruption of the $C A V 3$ gene can lead to different types of skeletal muscle disorders of autosomal dominant mode of inheritance such as familial hypertrophic cardiomyopathy (MIM 192600), hyperCKemia (MIM 123320), Tateyama type of distal myopathy (MIM 614321) and rippling muscle disease (MIM 606072), long QT syndrome 9 (MIM 611818), while disruption of the MTMR14 gene can lead to centronuclear myopathy (MIM 160150).

Chen et al. $[27,28]$ reported two prenatal cases with distal $3 p$ deletion. In the first case, cytogenetic and aCGH analysis showed a de novo deletion 3p25.3-pter, including a large number of genes that are involved in neurodevelopment disorders. The pregnancy was terminated at 24 weeks' gestation and fetal autopsy showed brachicephaly, micrognatia, short and thick nose, together with hypertelorism and low-set ears, which were also present in our case. The second prenatal case showed $3 p$ deletion accompanied with fetoplacental discrepancy [28].

A finding of isolated septated cystic hygroma during the first trimester of pregnancy is associated with chromosomal abnormalities in approximately $50.0 \%$ of cases, most commonly numerical chromosomal aberrations, e.g., monosomy X or trisomies 21, 13 and 18 [29]. Structural chromosomal abnormalities are considered as rare findings, detected in approximately $2.5 \%$ of such pregnancies [30]. The use of microarray analysis increases the probability of detection of pathogenic CNV to approximately $4.0 \%$ in cases of isolated NT and to $7.0 \%$ when additional malformations are present [31].
In conclusion, our case emphasizes the value of first trimester NT measurement as a screening method not only for the most common numerical chromosomal abnormalities, but also for rare structural rearrangements. Furthermore, the application of both conventional and molecular cytogenetic methods, are essential for detection and precise delineation of revealed aberrations, enabling detailed genetic counseling regarding present and future pregnancies.

Acknowledgments. Authors' contributions: M. Vulic, S.Z. Tomas and R.G. Juraski contributed to the identifica-tion of the local case; A. Vicic, F. Stipoljev and M. Logara performed the cytogenetic, FISH and aCGH analyses; M. Barbalic, R.G. Juraski and M. Logara drafted the manuscript; F. Stipoljev contributed significantly to the manuscript preparation. All authors read and approved the final manuscript.

Declaration of Interest. The authors report no conflicts of interest. The authors alone are responsible for the content and writing of this article.

\section{REFERENCES}

1. Bonaglia MC, Giorda R, Massagli A, Galluzzi R, Ciccone R, Zuffardi O. A familial inverted duplication/ deletion of $2 \mathrm{p} 25.1-25.3$ provides new clues on the genesis of inverted duplications. Eur J Hum Genet. 2009; 17(2): 179-186.

2. Aviram-Goldring A, Fritz B, Bartsch C, Steuber E, Daniely M, Lev D, et al. Molecular cytogenetic studies in three patients with partial trisomy $2 p$, including CGH from paraffin-embedded tissue. Am J Med Genet. 2000; 91(1): 74-82.

3. Lee C, Murray MF, Miron OM, Marsden D, Irons M, Wilkins-Haug LE, et al. Clinical picture: Multicolour karyotyping. Lancet. 2001; 357(9264): 1240.

4. Sperry ED, Schuette JL, van Ravenswaaij-Arts CMA, Green GE, Martin DM. Duplication 2p25 in a child with clinical features of CHARGE syndrome. Am J Med Genet A. 2016; 170A(5): 1148-1154.

5. Malmgren H, Sahlén S, Wide K, Cundvall M, Blennow E. Distal 3p Syndrome: Detailed molecular cytogenetic and clinical characterization of three small distal deletions and review. Am J Med Genet. 2007; 143(18): 2143-2149.

6. Moghadasi S, Haeringen A, Langerdonck L, Gijsbers ACJ, Ruivenkamp CAL. A terminal 3p26.3 deletion is not associated with dysmorphic features and intellectual disability in a four-generation family. Am J Med Genet A. 2014; 164A(11): 2863-2868. 
7. Chen C-P, Liu F-F, Jan S-W, Lin S-P, Lan C-C. Prenatal diagnosis of partial monosomy $3 p$ and partial trisomy $2 p$ in fetus associated with shortening of the long bones and a single umbilical artery. Prenat Diagn. 1996; 16(?): 270-275.

8. Wakita Y, Narahara K, Takahashi Y, Kikkawa K, Kimura S, Oda M, et al. Duplication of 2p25: Confirmation of the assignment of soluble acid phosphatise (ACP1) locus to 2p25. Hum Genet. 1985; 71(3): 259-260.

9. Roggenbuck JA, Fink JM, Mendelsohn NJ. Unique case of trisomy 2p24.3-pter with no associated monosomy. Am J Med Genet. 2001; 101(1): 50-54.

10. Tsurusaki Y, Koshimizu E, Ohashi H, Phadke S, Kou I, Shiina M, et al. De novo SOX11 mutations cause Coffin-Siris syndrome. Nat Commun. 2014; 5: 4011.

11. De Rocker N, Vergult S, Koolen D, Jacobs E, Hoischen A, Zeesman S, et al. Refinement of the critical 2p25.3 deletion region: The role of MYT1L in intellectual disability and obesity. Genet Med. 2015; 17(6): 460-466.

12. Lund ICB, Christensen R, Petersen OB, Vogel I, Vestergaard EM. Chromosomal microarray in foetuses with increased nuchal translucency. Ultrasound $\mathrm{Ob}$ stet Gynecol. 2015; 45(1): 95-100.

13. Thangavelu M, Frolich G, Rogers D. Partial duplication $2 p$ as the sole abnormality in two cases with anencephaly. Am J Med Genet A. 2004; 124A(2): 170-172.

14. Marlet L, Alix E, Till M, Raskin-Champion F, Attia $\mathrm{J}$, Boggio D, et al. Prenatal diagnosis of trisomy $2 \mathrm{p}$ due to terminal $2 p$ duplication including interstitial telomeric sequences. Cytogenet Genome Res. 2017; 153(3): 117-124.

15. Kuechler A, Zink AM, Wieland T, Lüdecke H-J, Cremer K, Salviati L, et al. Loss-of-function variants of SETD5 cause intellectual disability and the core phenotype of microdeletion 3p25.3 syndrome. Eur J Hum Genet. 2015; 23(6): 753-760.

16. Mattioli F, Schaefer E, Magee A, Mark P, Mancini $\mathrm{M}$, Dieterich K, et al. Mutations in histone acetylase modifier BRPF1 cause an autosomal-dominant form of intellectual disability with associated ptosis. Am J Hum Genet 2017; 100(1): 105-116.

17. Fernandez T, Morgan T, Davis N, Klin A, Morris A, Farhi A, et al. Disruption of contactin 4 (CNTN4) results in developmental delay and other features of 3 p deletion syndrome. Am J Hum Genet. 2004; 74(6): 1286-1293.

18. Suzuki-Muromoto S, Hino-Fukuyo N, Haginoya K, Kikuchi A, Sato H, Sato Y, et al. A case of $3 p$ deletion syndrome associated with cerebellar hemangioblastoma. Brain Dev. 2015; 2(2): 257-206.
19. Pohjola P, De Leeuw N, Penttinen M, Kääriäinen H. Terminal $3 p$ deletions in two families - Correlation between molecular karyotype and phenotype. Am J Med Genet A. 2010; 152A(2): 441-446.

20. Cuoco C, Ronchetto P, Gimelli S, Béna F, Divizia MT, Lerone M, et al. Microarray based analysis of an inherited terminal 3 p26.3 deletion, containing only the CHL1 gene, from a normal father to his two affected children. Orphanet J Rare Dis. 2011; 6: 12.

21. Cargile CB, Goh DL-M, Goodman BK, Chen X-N, Korenberg JR, Semenza GL, et al. Molecular cytogenetic characterization of subtle interstitial del(3) (p25.3-p26.2) in patient with 3p syndrome. Am J Med Genet. 2002; 109(2): 133-138.

22. Carvill GL, Jacinta M, McMahon JM, Schneider A, Zemel M, Myers CT, et al. Mutations in the GABA transporter SLC6A1 cause epilepsy with myoclonicatonic seizures. Am J Hum Genet. 2015; 96(5): 808-815.

23. Shuib S, McMullan D, Rattenberry E, Barber RM, Rahman F, Zatyka M, et al. Microarray based analysis of 3p25-p26 deletions (3p-syndrome). Am J Med Genet A. 2009; 149A(10): 2099-2105.

24. Robinson SW, Morris CD, Goldmuntz E, Reller MD, Jones MA, Steiner RD, et al. Missense mutations in CRELD1 are associated with cardiac atrioventricular septal defects. Am J Hum Genet. 2003; 72(4): 10471052.

25. Sotgia F, Minetti C, Lisanti MP. Localization of the human caveolin-3 gene to the D3S18/D3S4163/ D3S4539 locus (3p25), in close proximity to the human oxytocin receptor gene. Identification of the caveolin-3 gene as a candidate for deletion in $3 p-$ syndrome. FEBS Lett. 1999; 452(3): 177-180.

26. Peltekova IT, Macdonald A, Armour C. Microdeletion on 3 p25 in a patient with features of $3 p$ deletion syndrome. Am J Med Genet A. 2012; 158A(10): 2583-2586.

27. Chen C-P, Su Y-N, Chen C-Y, Su J-W, Chern S-R, Town D-D, et al. Pure partial monosomy 3p (3p25.3 $\rightarrow$ pter): Prenatal diagnosis and array comparative genomic hybridization characterization. Taiwan J Obstet Gynecol. 2012; 51(3): 435-439.

28. Chen C-P, Chen Y-Y, Chern S-R, Wu P-S, Su J-W, Chen W-L, et al. Prenatal diagnosis of a distal 3p deletion associated with fetoplacental chromosomal discrepancy and confined placental mosaicism detected by array comparative genomic hybridization. Taiwan J Obstet Gynecol. 2013; 52(2): 278-284.

29. Mack LM, Lee W, Mastrobattista JM, Belfort MA, Van den Veyver IB, Shamshirsaz AA, et al. Are first 
trimester nuchal septations independent risk factors for chromosomal anomalies? J Ultrasound Med. 2017; 36(1): 155-161.

30. Khalil A, Mahmoodian N, Kulkarni A, Homfray T, Papageorghiou A, Bhide A, et al. Estimation of detection rates of aneuploidy in high-risk pregnancy using an approach based on nuchal translucency and non-invasive prenatal testing: A cohort study. Fetal Diagn Ther. 2015; 38(4): 254-261.
31. Grande M, Jansen FA, Blumenfeld YJ, Fisher A, Odibo AO, Haak MC, et al. Genomic microarray in fetuses with increased nuchal translucency and normal karyotype: A systematic review and metaanalysis. Ultrasound Obstet Gynecol. 2015; 46(6): 650-658. 